\title{
Laser-induced plasma image velocimetry
}

\author{
Zhengjie Shi $^{1}$ (D) Yannis Hardalupas $^{1} \cdot$ Alex M. K. P. Taylor ${ }^{1}$
}

Received: 17 June 2018 / Revised: 3 November 2018 / Accepted: 12 November 2018 / Published online: 27 November 2018 (c) The Author(s) 2018

\begin{abstract}
A novel velocimetry method is proposed for point velocity measurement, which is based on tracking a laser-induced plasma in a flow. The plasma's behaviour is first analysed spatially, temporally and spectrally in quiescent air. The dependence of this technique on the delay time between subsequent plasma images and the processing methods are described. It is found that, for optimized operation of the technique in a turbulent air jet (exit diameter $10.0 \mathrm{~mm}$ from a $480 \mathrm{~mm}$ long pipe; with an averaged velocity of $50 \mathrm{~m} / \mathrm{s}$ at the jet exit resulting in Reynolds number of 34,000) with $100 \mu$ s time delay between plasma images, the systematic and random components of the velocity uncertainty are $-0.51 \mathrm{~m} / \mathrm{s}$ and $\pm 3.6 \mathrm{~m} / \mathrm{s}$ along the laser beam direction, and $1.25 \mathrm{~m} / \mathrm{s}$ and $\pm 0.86 \mathrm{~m} / \mathrm{s}$ along other directions perpendicular to the laser beam. These uncertainties are mainly caused by the asymmetric laser energy deposition during the formation of plasma, and the associated spatial resolution (in this realisation of the instrument) of $5 \mathrm{~mm}$. The mean velocity measurements in the turbulent air jet flow are consistent with the reported flow behaviour in the literature for mean velocity: the turbulent intensity of axial velocity fluctuations is comparable to those in the literature but difference arises due to the limited spatial resolution. This velocimetry method is an alternative to traditional tracer-based velocimetry methods, because it does not require 'seeding' of particles or other substances in the flow. It also has the ability to measure local gas mixture composition, using laser-induced breakdown spectroscopy approach, simultaneously with flow velocity, but this aspect is not explored in the current study.
\end{abstract}

Graphical abstract Concept of Laser-Induced Plasma Image Velocimetry (LIPIV) applied to a turbulent jet flow. The LIPIV technique measures the flow velocity vector by using the temporal displacement of the laser-induced plasma in a flowing fluid. For this reason, two sequential images of the plasma with time delay $\Delta t$ are used.

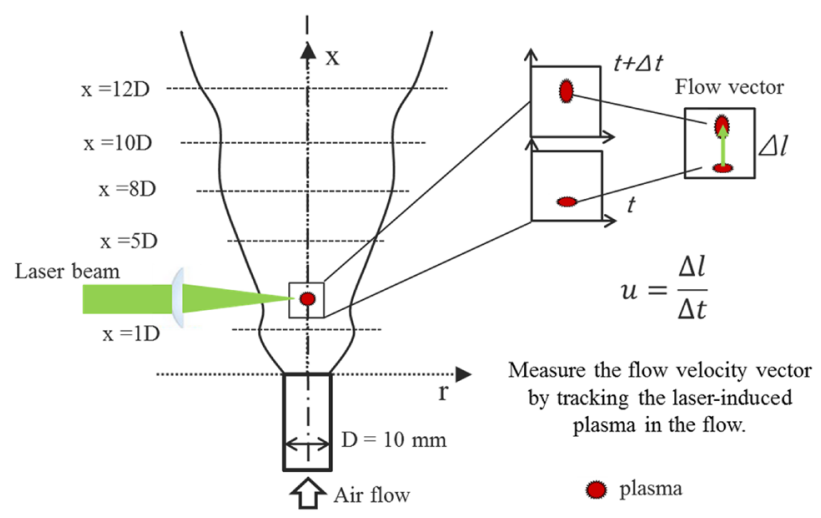

Zhengjie Shi

z.shi14@imperial.ac.uk

1 Department of Mechanical Engineering, Imperial College London, Exhibition Rd, London SW7 2AZ, UK

\section{Introduction}

The measurement of the local flow velocity characteristics frequently enhances the understanding of the physics of many flows, be these of technical or of research interest. Where possible, optical (frequently laser-based) velocimetry 
techniques are widely used because of their non-intrusive nature, of their ability to withstand harsh environments, their excellent accuracy and precision, and good spatial resolution. Some of these optical techniques are based on the tracking of either solid or liquid 'seeding' particles introduced into the flow, such as particle image velocimetry (PIV) (Stevens et al. 1998; Willert and Jarius 2002; Kiuchi et al. 2005; Troiani et al. 2009; Hong et al. 2013; Peterson et al. 2013) or laser Doppler velocimetry (LDV) (Sislian et al. 1988; Edwards and Rudoff 1990; Van Maaren and De Goey 1994; Van Maaren et al. 1994; Lyn et al. 1995; Tropea 1995). In some cases, the introduction of seeding particles with sufficiently low inertia to follow the flow faithfully, and at adequate concentration, is feasible. In contrast, in other cases the introduction of seeding particles is either undesirable (because the particles may damage the flow under consideration, e.g., settling screens in wind tunnels, gas turbine combustors, the liners of internal combustion engines) or not possible (because, for example, in some confined flows, the particles may deposit too heavily on the surfaces of the windows that may be necessary for optical access or because of the large flow rates involved).

Thus, several laser-based non-intrusive velocimetry techniques have been developed. For example, filtered Rayleigh scattering (FRS) measures the Doppler shift of molecularly scattered light to determine the gas flow velocity (Forkey et al. 1996). The technique is best applied to relatively high-speed flows, where the Doppler shift is large enough to measure accurately. In molecular tagging velocimetry (MTV), a specific molecular marker is written into the gas and the velocity is measured by computing the marker's displacement over a known delay time (Stier and Koochesfahani 1999; Lempert et al. 2002; Hu and Koochesfahani 2006). Some of the MTV methods also add tracer molecules into gas flows, which is similar to the concept of particlebased methods. Examples are the phosphorescence of biacetyl (Stier and Koochesfahani 1999) or acetone (Lempert et al. 2002) added into nitrogen flows. In other MTV methods, available gas species in the flow are used as tracers or markers. For example, ozone tagging velocimetry (OTV) produces ozone from oxygen via the photodissociation process; hydroxyl tagging velocimetry (HTV) produces $\mathrm{OH}$ via dissociation of water vapour (Pitz et al. 2000; Ribarov et al. 2004). Similarly, Raman excitation combined with laserinduced electronic fluorescence (RELIEF) uses a nonlinear stimulated Raman process to produce a tag line of vibrationally excited oxygen $\left(\mathrm{O}_{2}\right)$, whose enhanced fluorescence excitation strength (over that of ground-state $\mathrm{O}_{2}$ ) reveals the displacement of the tag when the flow field is subsequently irradiated by an ultraviolet (UV) laser (Miles et al. 1987). Air photolysis and recombination tracking (APART) is based on laser-induced fluorescence of NO molecules from dissociation of oxygen (Sijtsema et al. 2002). These MTV methods rely on the presence of large signal intensities and a sufficiently long lifetime of the tagged molecules. A high power UV laser is generally necessary for MTV and the lifetime of the tracers depends on the quenching caused by certain species or pressure (Pitz et al. 2000). Other velocimetry techniques, such as laser-induced thermal acoustics (Hart et al. 2000) or magnetic resonance velocimetry (Elkins et al. 2003; Elkins and Alley 2007), have high experimental complexity. In this work, we investigate another technique which does not require the introduction of seeding particles.

Laser-induced breakdown spectroscopy (LIBS: Stavropoulos et al. 2005; Majd et al. 2011; Lee et al. 2016; Shi et al. 2016) is based on a pulsed laser beam, of sufficiently high energy, focused to a small volume of a gas to generate a local laser-induced plasma in the flow. The light emitted from the laser-induced excited atoms during their relaxation process is recorded and analysed to determine the elemental compositions of the sampled substances qualitatively and quantitatively (Molina et al. 2005; Ferioli and Buckley 2006; Yaroshchyk et al. 2006; Joshi et al. 2009; Kotzagianni et al. 2014; McGann et al. 2015). Kawahara et al. (2006) have reported on the spatial, spectral and temporal behaviour of laser-induced plasma in air; and Dors and Parigger (2003) have simulated the fluid dynamic behaviour of the laserinduced plasma in air, which agreed well with experimental investigations. The emitted light from the LIBS plasma can also be recorded by a camera to identify its location at successive times and this information can be used to evaluate the velocity field.

A novel measurement technique for velocimetry in fluid flows is proposed and developed in the current paper, which is based on LIBS and hence does not require the introduction of either 'seeding' particles or substances in the flow. Instead of tracking the velocity of 'seeding' particles, the convected laser-induced plasma by the flow is tracked as a function of time. The method does not depend on the presence of any species, such as tracers or markers, in the gas before measurements, since the laser-induced plasma can be initiated in all gases with appropriate laser energy. The flow velocity vector is estimated from the displacement of the plasma within a time $\Delta t$, as shown in Fig. 1 . To the extent that a plasma is created in the flow with a finite lifetime, of order millimeter dimensions together with an associated localised velocity field associated with the plasma, this method is intrusive.

The paper is structured as follows. The behaviour of laser-induced plasma in air is first investigated and analysed. The laser-induced plasma image velocimetry (LIPIV) technique is then evaluated and optimized in quiescent air, so that the uncertainties can be quantified. The influences of the delay time between recorded plasma images and processing methods are evaluated. Then, LIPIV is applied to measure the velocity in a turbulent round jet flow, which 


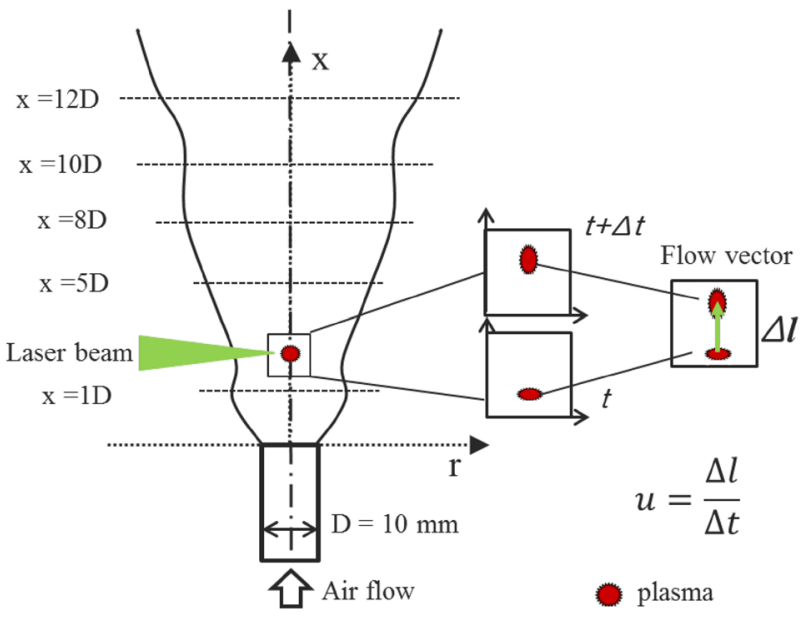

Fig. 1 Concept of laser-induced plasma image velocimetry (LIPIV) applied to a turbulent jet flow. The LIPIV technique measures the flow velocity vector using the temporal displacement of the laserinduced plasma in a flowing fluid. For this reason, two sequential images of the plasma with time delay $\Delta t$ are used

has a well-known velocity field (Mi et al. 2007; Aziz et al. 2008; Uchiyama et al. 2013). The measured flow velocity development along the jet centreline, the self-similar mean velocity radial profiles and the radial profiles of turbulent intensity are used to evaluate the accuracy of the technique. The advantages and limitations of the LIPIV technique are finally summarized.

\section{Velocimetry method}

The local fluid velocity vector convects the induced plasma during its lifetime. If the plasma is recorded at two instants separated by a delay $\Delta t$, its displacement due to the flow field can be quantified and, as a consequence, an estimate of the flow velocity is obtained. For the LIPIV method, the measured velocity $u_{\mathrm{m}}(x, t)$ can be decomposed as a linear model:

$u_{\mathrm{m}}(x, t)=u(x, t)+u^{*}(x, t)$

In Eq. (1), $u$ is the velocity component of the flow field and $u^{*}$ is a velocity component originated by the laserinduced plasma, which represents an uncertainty. The linear model of Eq. (1) assumes that the uncertainty of the LIPIV is independent of the flow and additional studies in swirl-stabilised flows (Chap. 7 of Shi 2018), so far support this assumption. This study will form the subject of a future publication and provide evaluation of additional uncertainties. The contributions to $u^{*}$ in Eq. (1) include:

(a) The effects of the asymmetric pressure wave in the flow in directions perpendicular to the laser beam's axis, generated by the laser-induced plasma, which may disturb the local flow field.

(b) The fluctuations of the laser beam shape, camera sensitivity and buoyancy effects on the measurement of the plasma's displacement.

Therefore, the underlying mean flow velocity and the time-averaged root mean square of the velocity fluctuations about the mean, $u_{\text {rms }}$ can be calculated according to Eqs. (2) and (3), after removing the corresponding laser-induced components.

$\bar{u}=\overline{u_{\mathrm{m}}}-\overline{u^{*}}$

$u_{\mathrm{rms}}=\sqrt{u_{\mathrm{m}, \mathrm{rms}}^{2}-u_{\mathrm{rms}}^{* 2}}$

In a turbulent flow, these moments are estimated by ensemble averaging measurements over a number of laserinduced plasma realisations. It is noted that the current work investigates the performance of the LIPIV technique in a simple jet air flow. In more complex fluid flows, where high out-of-plane motions and non-uniform temperature and pressure fields are present, the feasibility of the LIPIV requires further investigation. An evaluation of the uncertainties of the LIPIV technique in non-reacting and reacting swirling flows is available in Chap. 7 of Shi (2018).

\section{Experimental details}

Figure 1 shows the investigated flow configuration, an air jet air exhausting from a $480 \mathrm{~mm}$ long straight pipe with $10.0 \mathrm{~mm}$ internal diameter. In this way, the pipe flow develops over 48 diameters, which ensures fully developed pipe flow at the exit. The pulsed laser beam was generated by the Surelite III Q-switched Nd:YAG laser, delivering pulses of $532 \mathrm{~nm}$ light with a nominal 3-5 ns pulse width at a $10 \mathrm{~Hz}$ frequency. The pulse energy was kept at $125 \mathrm{~mJ}$. For the measurement of the plasma image and spectra temporal evolution, Fig. 2a shows the optical arrangement of the LIBS system, which comprised a $35 \mathrm{~mm}$ focal length UV fused silica plano-convex lens to collect the emitted light from the plasma at right angles and direct it to an optical fibre (Edmund $1000 \mu \mathrm{m}$ diameter, $180-1150 \mathrm{~nm}$ ). The fibre transmitted the light to a spectrograph (Mechelle 5000 with a $50 \times 25 \mu \mathrm{m}$ input slit) and an ICCD camera (Andor DH534-18F-03). Together, the combination of the ICCD and the spectrograph measured over wavelengths from 200 to $850 \mathrm{~nm}$ with spectral continuity (Spectral resolution: $0.125 \mathrm{~nm}$ at $500 \mathrm{~nm}$ ). A second ICCD camera (Andor DH534-18F-03, field of view $11.5 \times 11.5 \mathrm{~mm}^{2}$, resolution $=25.3 \mu \mathrm{m} / \mathrm{pixel}$ ) was used to record the image of the plasma. The delay time relative to the trigger signal, and 


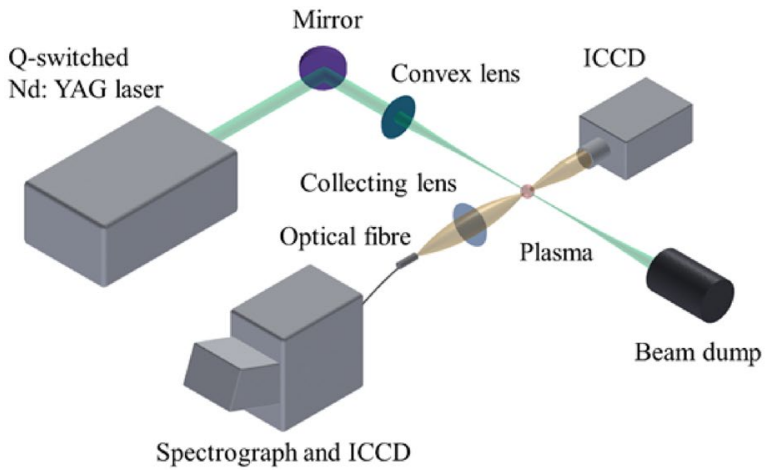

(a)

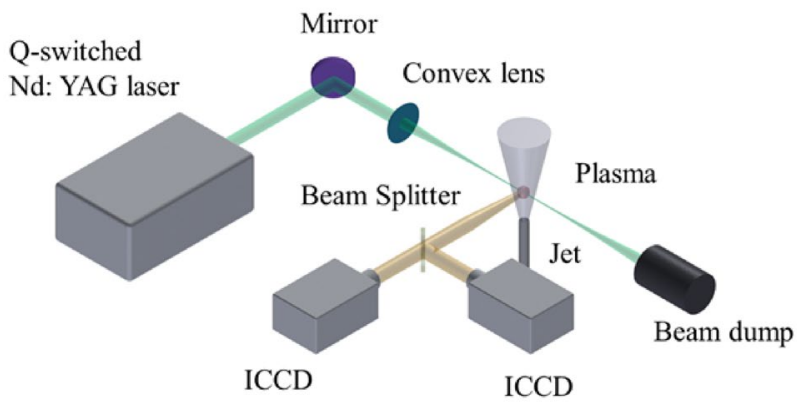

(b)

Fig. 2 Schematic diagram of the LIBS (a) and LIPIV (b) experiment setup

the aperture time of the ICCD and spectrometer were controlled through a multi-channel digital delay pulse generator (NI PCI-6602) that controlled the pulsed laser and ICCDs. A Newport 6035 Mercury spectral lamp and a Hamamatsu L7810 Xenon lamp were used to calibrate the wavelengths and the relative intensity, respectively.

Figure $2 \mathrm{~b}$ shows the optical arrangement of the LIPIV system, with differences from that of the LIBS arrangement as follows. Two cameras were used to record the two plasma images, one immediately after the initiation of the plasma and the other after the plasma has been convected by the flow after the chosen time delay. The set-up of the first camera, in particular, is designed to counteract uncertainties due to variation of the initial location of the plasma in the flow, which can be affected by factors such as beam steering and influences caused by the flow, material properties, and the spatio-temporal profile of each laser pulse. Both ICCD cameras (Andor DH534-18F-03) with Nikon MicroNIKKOR AI $105 \mathrm{~mm} \mathrm{f} / 4$ lenses had a field of view $26 \times 26$ $\mathrm{mm}^{2}$ with resolution of $25.3 \mu \mathrm{m} /$ pixel. An uncoated pellicle acted as a beam splitter with reflection-to-transmission ratio of 8:92 for wavelengths between $400 \mathrm{~nm}$ and $2400 \mathrm{~nm}$. Due to the high intensity of the plasma emissions at early times after the laser pulse, reflected light was used to record the first image of the plasma while the transmitted light as used

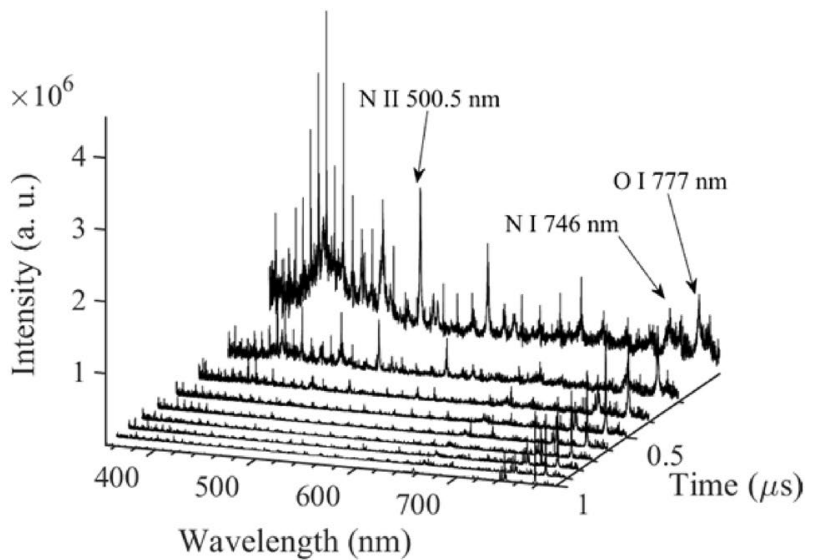

(a)

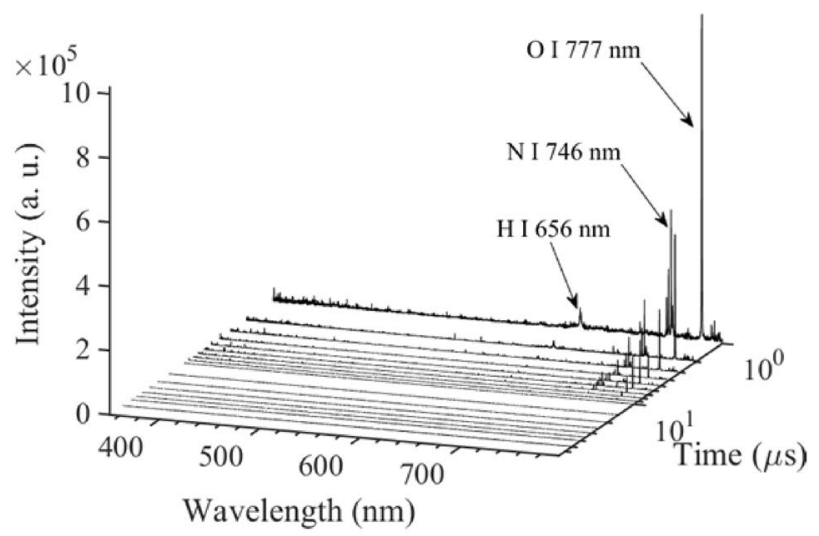

(b)

Fig. 3 LIBS spectra from air as a function of time delay after the laser pulse a $0.1-1 \mu$ s and b $1-100 \mu$ s

for the second, low intensity image of the plasma. In the experiment, the temperature of the air supply was at room temperature $\left(15^{\circ} \mathrm{C}\right)$. The air mass flow rate was controlled using Bronkhorst digital mass flow controllers (accuracy: $\pm 0.5 \%$ reading, $\pm 0.1 \%$ full scale).

\section{Results and discussion}

\subsection{Spatial, temporal and spectral evolution of plasma in air}

For a Q-switched Nd: YAG laser with nanosecond pulse width, the plasma spectra were initially dominated by continuous light caused by the Bremsstrahlung effect and recombination radiation (Miziolek et al. 2008). Figure 3a shows that during time delays of $0-1 \mu$ s after the laser pulse, the plasma was created with strong continuous background light. These spectrally continuous emissions contained little 
information about the plasma composition. During this process, the high energy level ionic emissions, such as $\mathrm{N}$ II at $463.0 \mathrm{~nm}$ and N II at $500.5 \mathrm{~nm}$, co-existed with the continuous emissions which decayed within about $1 \mu$ s. Low energy level, neutral atomic emission lines, such as N I (746 nm) and O I (777 nm), emerged as the continuum emission weakened gradually in this period. Figure $3 \mathrm{~b}$ shows that, $1 \mu \mathrm{s}$ after the laser pulse, the spectral intensity decreased and the continuum emission vanished for time delays between 1 and $10 \mu \mathrm{s}$. The neutral atomic emissions dominated the spectra until $10 \mu$ s. Subsequently, as the plasma continued to cool down, the atoms recombined to form new molecules. After around $10 \mu$ s, the spectrometer was not sensitive enough to capture the spectra and only the ICCD (at high intensifier level) was able to record the plasma image. Theoretically, at this time, the plasma was already cool and only molecules existed with weak emissions. Then, the plasma evolved from a hot spot into some scattered parts and dispersed widely in the field. The spectral evolution of the plasma of Fig. 3 is important for the selection of the spectral characteristics of the camera for LIPIV measurements. The spectra, before 1 $\mu \mathrm{s}$, were broadband and, after $1 \mu \mathrm{s}$, the spectral emission was mainly in the infrared region. Since the plasma image was recorded after $1 \mu \mathrm{s}$, it was better to capture the plasma image with a camera that was sensitive to infrared light. In addition, the spectral emission can also be used to detect the local chemical composition of the gas, based on the LIBS technique. In this way, LIBS gas composition measurements can be obtained simultaneously with the plasma displacement measurements to provide flow velocity using LIPIV. LIPIV and LIBS measurements of flow velocity and local gas composition have been demonstrated in a swirl-stabilised flame under atmospheric conditions, with and without reaction (Chap. 7 of Shi 2018), although additional equipment to that presented here is required to do so.

The laser-induced plasma decay process in a gas has been studied both experimentally and numerically by several researchers (Kravchik and Sher 1994; Akram 1996; Morsy and Chung 2002; Prager et al. 2003; Bradley et al. 2004). The measured temporal evolution process of the laserinduced plasma is shown in Fig. 4 with a schematic of the same in Fig. 5. When the plasma is created in a gas, a shock wave moves outwards, while the temperature and pressure of the inner gas region begin to decrease with time. The plasma's size increases over the first $5 \mu$ s after its creation. As shown in Fig. 6, the rarefaction wave produces a pressure gradient towards the plasma centre. The gas with lower density is accelerated more by the pressure gradient than the gas of higher density due to Taylor instability (Taylor 1950). As a consequence, the hot gas on the right in Fig. 6 moves more persistently inwards than the gas on the left (which is less dense) and subsequently the gas on the right reaches a higher velocity, which decays rapidly. This velocity

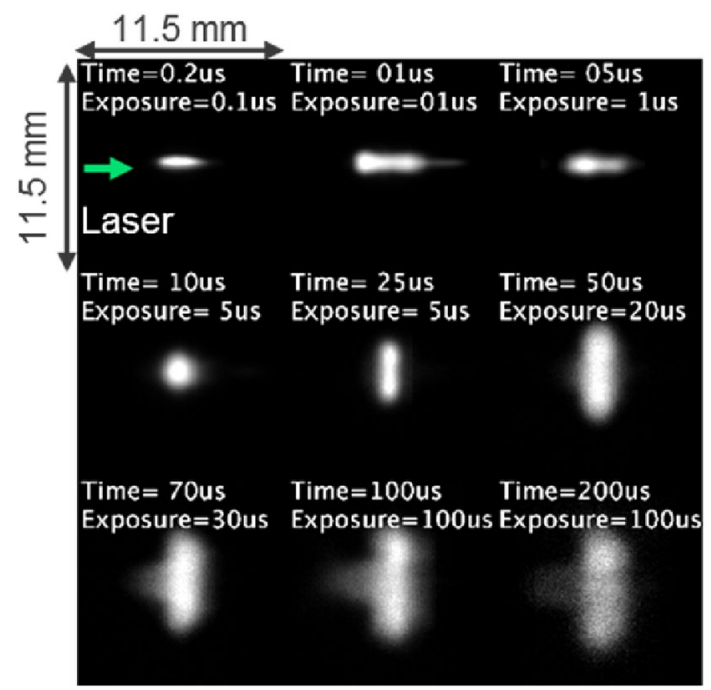

Fig. 4 Measured sequences of the temporal development of laserinduced plasma, generated by a laser pulse of $125 \mathrm{~mJ}$ in air

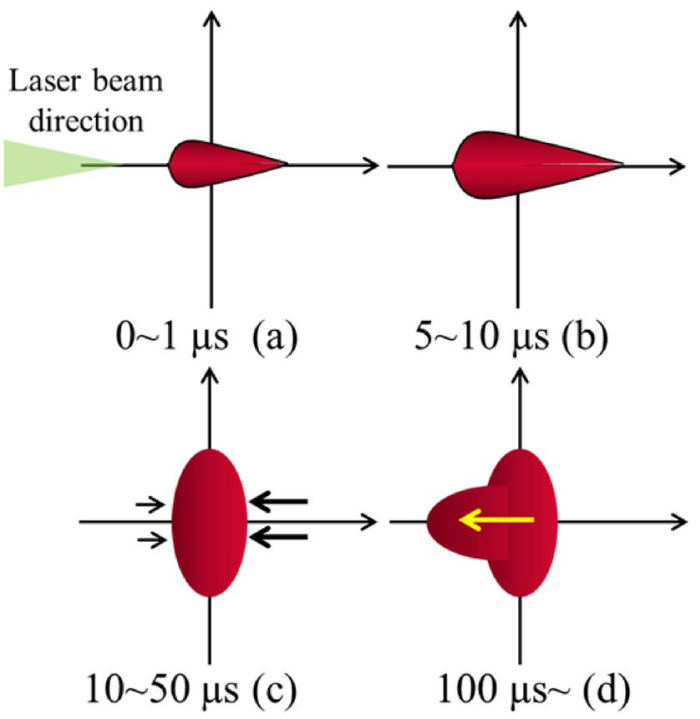

Fig. 5 Typical temporal evolution process of nanosecond laserinduced plasma (Dors and Parigger 2003; Bradley et al. 2004)

difference between the laser-induced plasma gases results in vortex generation, which is more persistent at the front of the laser beam rather than at the region behind the focal point. The subsequent collapse of the two opposed jet-like fronts generates vortices that form a toroidal shape around the optical axis of the laser beam after about $5 \mu$ s after the laser pulse (Morsy and Chung 2002). This toroidal flow structure deforms the ellipsoidal shape of the plasma, so that its principal axis eventually becomes orthogonal to the laser beam with time (see Fig. 5b, c). Because the toroidal structure is not symmetric, the laser-induced flow velocity along the laser beam axis is, at a later times, towards the laser source 


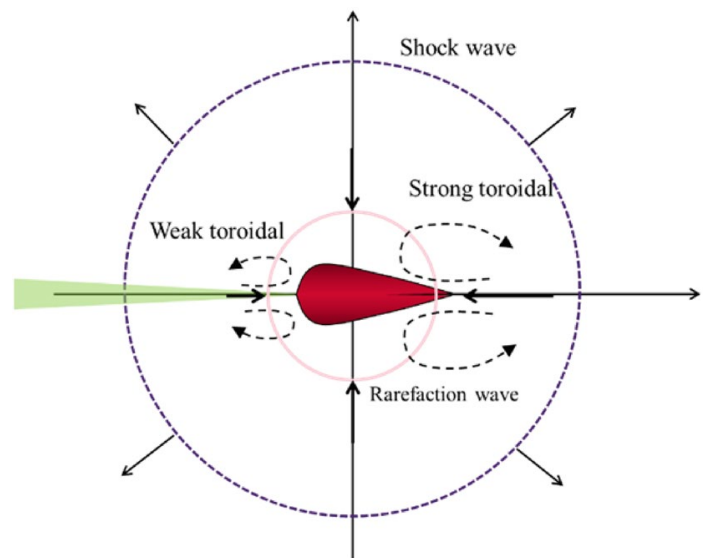

Fig. 6 Relative position of shock and rarefaction waves and initiating plasma kernel at a time of around $5 \mu$ s after the laser pulse (Bradley et al. 2004)

and generates a new, 'third', lobe in that direction, as shown in Fig. 5d. Therefore, due to the asymmetric energy distribution in the gas and subsequent asymmetric expansion, the asymmetric collapse of the plasma leads to the collapsing front moving towards the laser beam direction. (some researchers attribute the generation of the third lobe to the gas movement 'upstream' from the focal point due to the ionized front of the plasma propagating towards the laser at the early stages of the laser-induced plasma (Raizer 1977). However, this movement is not expected to last for a long time after the end of the laser pulse. Therefore, most of the studies attribute this process to the over-expanded region, explained above). Since these plasma kernel structures are developed due to the vertical motions generated by the interaction of the pressure field and the flow, caused by the asymmetric deposition of laser energy along the laser beam direction, the behaviour of the plasma is sensitive to the initial pressure field and, therefore, the laser pulse energy. Variations of the laser pulse energy can lead to variations of the temporal evolution of the plasma that leads to the variation of the velocity measured in quiescent flow, reported in Sect. 4.2. These processes have been analysed by different researchers using various techniques, including LIF, shadowgraphy and imaging (Dors and Parigger 2003; Bradley et al. 2004; Mulla et al. 2016) and are similar to the observed flow for plasmas induced by electrical sparks (Kono et al. 1989).

Therefore, the asymmetric laser energy deposition and the ionization effects determine the local fluid properties in the post-breakdown period and the flow pattern is characteristic of the laser-induced plasma decay. This induced flow will be considered in the subsequent development of the LIPIV technique.

Figure 7 shows the plasma size temporal evolution in air. Both the horizontal length $L_{x}$ and the vertical diameter

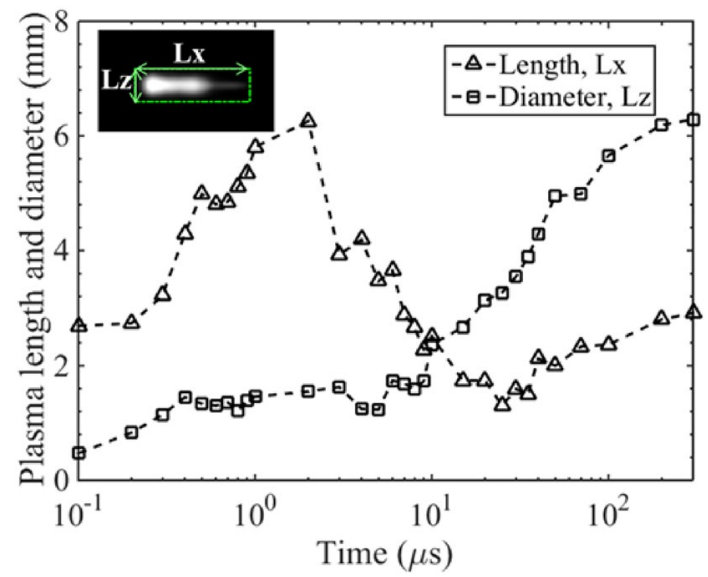

Fig. 7 Plasma length $L_{x}$ (triangles) and diameter $L_{z}$ (squares) as a function of time after a laser pulse with $125 \mathrm{~mJ}$ pulse energy in air. $x$ and $z$ coordinates are along and normal to the direction of the laser beam, respectively. $L_{x}$ and $L_{z}$ represent the dimensions along the $x$ axis (horizontally) and $z$ axis (vertically)

$L_{z}$ increased with time after the laser pulse and began to decrease after around $1-2 \mu$ s but, after $20 \mu$ s, both the $L_{x}$ and $L_{z}$ began to grow continuously again until the plasma disappeared due to the void collapse in the plasma. The lengths of $L_{x}$ and $L_{z}$ jointly determine the size of the probe volume at different times. For a laser pulse with energy of $125 \mathrm{~mJ}$ in air, the probe volume grew to around $2.5 \mathrm{~mm} \times 5 \mathrm{~mm} \times 5 \mathrm{~mm}$ at time $100 \mu$ s after the laser pulse initiation, which is an important consideration for the spatial resolution of the laser-induced plasma velocimetry technique. Although larger size plasma has the advantage that it leads to higher signal-to-noise ratio (SNR) of the detected spectral intensities, which means that temporal tracking of the plasma for velocimetry measurements is easier, at the same time larger size plasma reduces the spatial resolution of the velocity measurement in a flow. Therefore, there is a need for a compromise between the SNR of LIBS spectral intensity and spatial resolution of the LIPIV technique. Figure 7 shows that, for time delay between 50 and $100 \mu$ s, the dimensions of the plasma volume are fairly constant and, therefore, this appears to be an appropriate compromise for plasma displacement measurements for LIPIV. It is noted that this information is for air at atmospheric and therefore, for different gases and pressures, a similar optimisation is required to ensure the best selection of the time delay between the two plasma images.

\subsection{LIPIV: accuracy and precision due to laser-induced velocity in a quiescent gas}

In quiescent air, the flow velocity is zero and the resulting velocity uncertainty $u^{*}$ of Eq. (1) can be evaluated. The effects of three image processing methods and timing 
settings for the plasma recordings are evaluated and optimized below.

\subsubsection{Processing method I: bounding box centre}

The first image processing method for tracking the location of the laser-induced plasma as a function of time after the laser pulse is based on a "bounding box centre" approach (The Mathworks Inc. 2014).

The pair of recorded plasma images was first converted into binary format. The smallest rectangle containing the plasma was identified on the image using custom code (developed in MATLAB ${ }^{\circledR}$ ). The concept underlying this approach is that the smallest rectangle containing the plasma may be insensitive to plasma shape and noise present on the image. When using this method, the first image was obtained $1 \mu \mathrm{s}$ after the laser pulse initiation, which helped locate the plasma kernel. Then, after a delay time ranging from 25 to $300 \mu$ s, the second image was recorded. A moderate delay time (typically $50-100 \mu \mathrm{s}$ ) between the two plasma images allowed a plasma displacement of the order of $1 \mathrm{~mm}$, which was large enough to reduce the uncertainty and ensure proper temporal and spatial resolution. The centre of the bounding rectangle can be identified directly from the intersection of the two diagonals of the rectangle (green spot in Fig. 8a). The centroid was then tracked over the time delay between the two images, the displacement was quantified and the two-dimensional velocity vector was calculated in the "horizontal" component, which was along the laser beam axis, and the "vertical" component, which was normal to the axis of the laser beam. The deviation of the measured velocities from the expected value of zero in the ambient represents the contribution of the flow induced by the laserinduced plasma and uncertainties related to the image processing method.

The size of the bounding boxes in the images of Fig. 8a are $5 \mathrm{~mm} \times 2 \mathrm{~mm}$ and $3 \mathrm{~mm} \times 5 \mathrm{~mm}$ for times after the laser pulse initiation of 1-2 $\mu$ s and 50-60 $\mu$ s, respectively, and this sets the spatial resolution of the LIPIV. It can be seen that, during the time windows $25-300 \mu$ s for LIPIV, both the width and height of the plasma continued to grow with the time delay. The spatial resolutions of the geometric centre and weighted centroid processing methods, described below, are the same as the bounding box method. A compromise between plasma displacement (affecting the accuracy of the velocity measurement) and spatial resolution of the LIPIV technique should be considered.

\subsubsection{Processing method II: geometric centre}

The second method is based on evaluating the geometric centre of the plasma. Figure $8 \mathrm{~b}$ shows the geometric centre of the plasma (red spot) at the two time delays relative

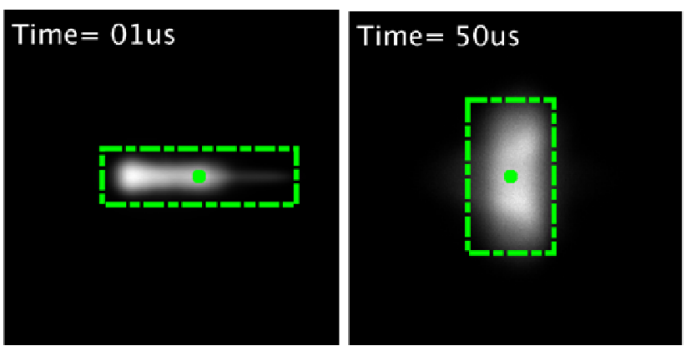

(a)

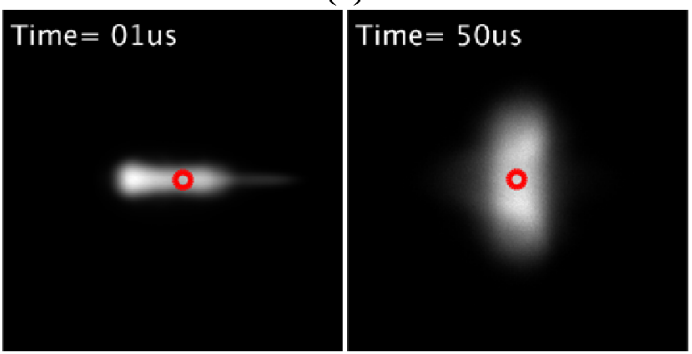

(b)
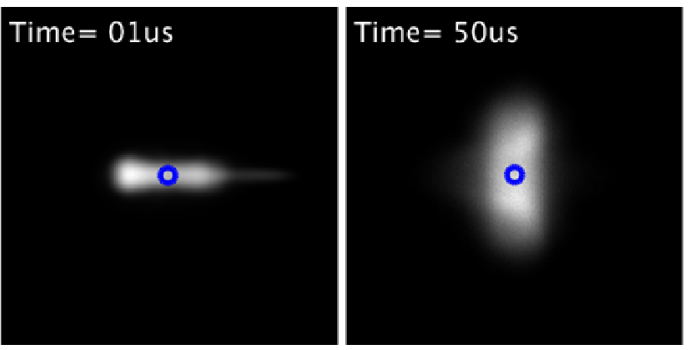

(c)

Fig. 8 Image processing methods for the identification of the centre of the recorded plasma at times $1 \mu$ s and $500 \mu$ s after the laser pulse. a Bounding box centre; $\mathbf{b}$ geometric centre; $\mathbf{c}$ weighted centroid. The laser beam was incident from the left hand side and all the images span $11.5 \mathrm{~mm}$ horizontally and $11.5 \mathrm{~mm}$ vertically. The identified plasma centre is green for the bounding box centre method, red for the geometric centroid method and blue for the weighted centroid

to the laser pulse initiation. This is determined following similar processing procedures as for the bounding box centre method, except for the determination of the location of the centre of mass (intensity) of the binary plasma on the image.

\subsubsection{Processing method III: weighted centroid}

The third method was based on a weighted plasma centroid approach: the approach differs from the two previous processing methods in that it calculates the weighted centre of the plasma directly from the greyscale images of the plasma intensity, rather than binarised images. In this way, the spatial distribution of the intensity emitted by the plasma influences more directly the identified centre rather than the other two methods. In Fig. 8c, the weighted centroid results are shown in blue symbols.

Figure 9 shows the standard deviation of the velocity fluctuations of the laser-induced plasma as a function of the 


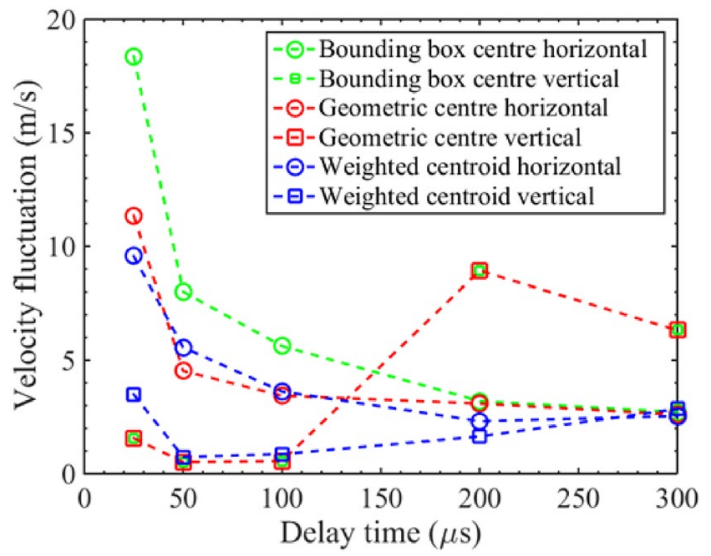

Fig. 9 Standard deviation of velocity fluctuations in quiescent air as a function of time delay between the two plasma images based on 300 laser shots using the three processing methods of Fig. 8. Note that the first plasma image is always recorded at time $1-2 \mu$ s after the initiation of the laser pulse (circles denote horizontal rms velocity and squares the vertical rms velocity)

delay time between the recorded plasma images in quiescent air. The optimum time delay is the one that leads to the minimum standard deviation of velocity fluctuations for both velocity components: delay times $50 \mu$ s and $100 \mu$ s between the two plasma images (note that the first plasma image is recorded always at time $1-2 \mu$ s after the initiation of the laser pulse) represent this optimum. The horizontal and vertical velocity fluctuations measured with all the processing methods were less than $5 \mathrm{~m} / \mathrm{s}$ and $1.0 \mathrm{~m} / \mathrm{s}$, respectively, for 100 $\mu$ s delay time. Because the weighted centroid method has smaller velocity uncertainty than the other methods in the quiescent field, this method was used for the following flow velocity measurements.

Figure 10 presents the histograms of the instantaneous velocity measurements for horizontal (along the laser beam direction) and vertical (normal to laser beam direction) components with time delays of $50 \mu$ s and $100 \mu \mathrm{s}$ using the weighted centroid image processing method. In quiescent air, the true flow velocity was zero so that the only existing component of Eq. (1) was $u^{*}$. Therefore, laser-induced motion results in systematic ('accuracy') and random ('precision') components to the uncertainty. The results for $100 \mu$ s time delay between the two plasma images show that the horizontal direction measurement $\mathrm{had} \pm 3.6 \mathrm{~m} / \mathrm{s}$ random uncertainty and the vertical direction had $0.86 \mathrm{~m} / \mathrm{s}$ random uncertainty. The $100 \mu$ s systematic uncertainty $\overline{u^{*}}$ was $-0.51 \mathrm{~m} / \mathrm{s}$ horizontally and $1.25 \mathrm{~m} / \mathrm{s}$ vertically, which can be removed from the actual flow velocity measurements using Eq. (1). In the turbulent jet flow experiments, presented in the next section, the velocity component of the jet flow was negligible in the direction normal to the jet axis compared to the
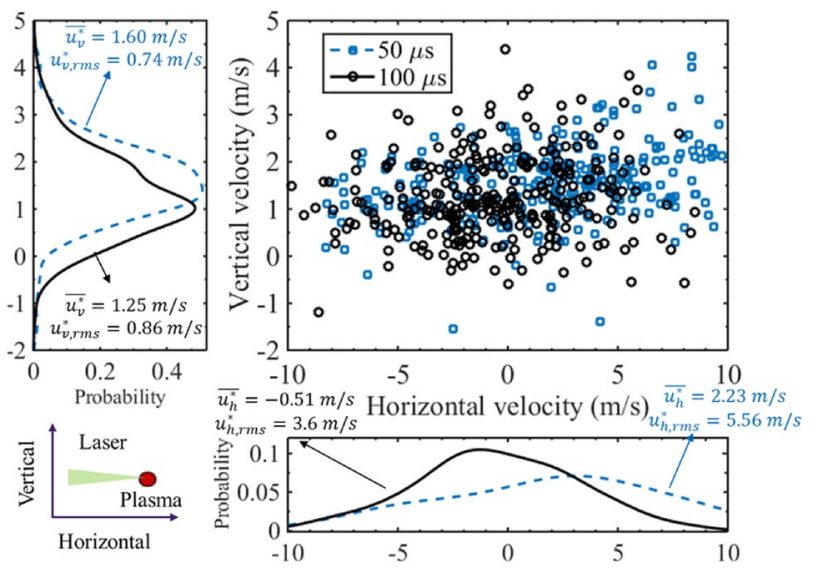

Fig. 10 Scatterplot of 300 instantaneous LIPIV measurements in quiescent air for $50 \mu$ s and $100 \mu$ s delay times between the recorded plasma images using the weighted centroid method (squares for 50 $\mu$ s and circles for $100 \mu$ s time delays). The vertical and the horizontal velocity components are normal to, and along, the laser beam axis, respectively. The upper left and bottom right graphs are histograms of the vertical and horizontal velocity, respectively

velocity along the jet axis. In terms of the spatial resolution of the technique, Fellouah and Pollard (2009), for example, have estimated that the smallest (Kolmogorov) length scale of the flow in a turbulent jet with Reynolds number $6000-10,000$ is of the order of $10^{-1} \mathrm{~mm}$ within the downstream locations that we are considering here. Without considering differences between their experiment and ours too deeply, it is certain that the plasma size is at least one order of magnitude larger than the Kolmogorov scale. This indicates that LIPIV velocity measurement has limited spatial and temporal resolution in the jet flow presented below. It may be possible to reduce the size of the plasma by optimising the delivered laser energy.

A note is required on the dynamic range of LIPIV velocity measurement, which mainly depends on the field of view of each camera. In this work, the field of view was $26 \mathrm{~mm} \times 26 \mathrm{~mm}$ with a camera resolution of $1024 \times 1024$ pixels and, therefore, the minimum displacement that the camera could measure was about $25 \mu \mathrm{m}$. For 50 and 100 $\mu$ s time delays, the corresponding velocity resolutions are $0.5 \mathrm{~m} / \mathrm{s}$ and $0.25 \mathrm{~m} / \mathrm{s}$, respectively. If the size of the recorded plasma is taken into account, and assuming that the plasma is induced at the centre of the field of view, the movement of plasma could be up to $10 \mathrm{~mm}$ in each of the directions. Therefore, the dynamic ranges for the velocity were $\sim 200 \mathrm{~m} / \mathrm{s}$ and $\sim 100 \mathrm{~m} / \mathrm{s}$ for $50 \mu$ s and $100 \mu$ s time delay, respectively, along each direction for the current experiment. The dynamic range of LIPIV can be adjusted by changing the field of view and the camera resolution. 


\subsection{Turbulent jet axial velocity measurements}

The streamwise ( $x$ direction) mean velocity distribution along the jet axis is measured for an averaged velocity of $50 \mathrm{~m} / \mathrm{s}$ at the jet exit with diameter $D$, resulting in Reynolds number of 34,000. Figure 11 shows that the mean flow velocity for $50 \mu$ s and $100 \mu$ s time delays between plasma images along the centreline axial direction is in good agreement with theoretical expectations. The mean differences between the experimental velocity results and theoretical curve were $-2.0 \mathrm{~m} / \mathrm{s}$ and $-0.1 \mathrm{~m} / \mathrm{s}$ for $50 \mu$ and 100 $\mu$ s delay times, respectively. A "potential core like" (the inverted commas denoting that a true potential core does not exist for a fully developed flow) region was observed up to $x / D<3$. This centreline velocity was proportional to $x^{-0.5}$ distance from nozzle exit. Compared to jet flows generated downstream of either a contraction or sharp-edge orifice pipes, the jet flow from long pipes, as used here, has the lowest velocity decay rate along the centreline (Mi et al. 2001). After $x / D=10$, the decay rate for time delay of $50 \mu \mathrm{s}$ was lower than the theoretical curve. Compared with $100 \mu$ s time delay, the $50 \mu$ s time delay measurement resulted in a smaller plasma displacement, leading to larger uncertainty especially for the low centreline velocity at such large values of $x / D$. The difference also indicated the imperfection of the linear model in Eq. (1) for low velocity, which needs further assessment. The measured mean velocity for $100 \mu$ s time delay is in better agreement with the theory.

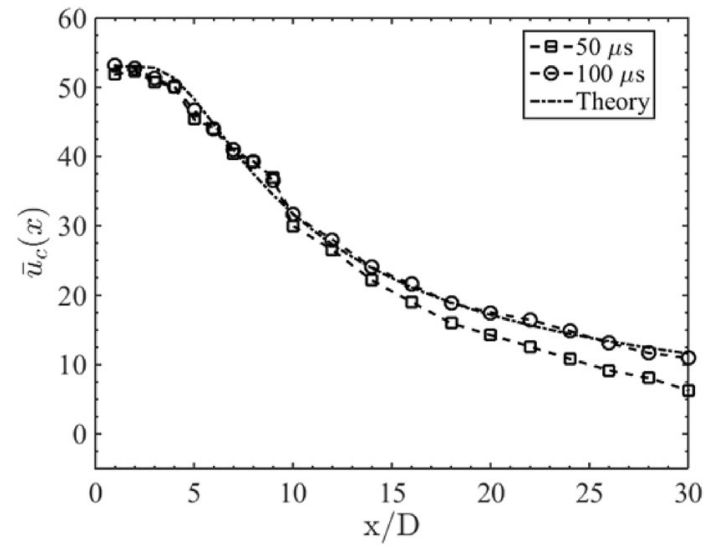

Fig. 11 Mean axial velocity for different time delays (squares for 50 $\mu$ s and circles for $100 \mu$ s time delays) between the recorded plasma images along the jet centreline averaged over 300 instantaneous velocity measurements at each point. The theoretical curves were plotted using the simple expression (Alexander et al. 1953):

$\frac{\bar{u}_{\mathrm{c}}(x)}{\bar{u}_{\mathrm{c}}(0)}=\sqrt{1-\mathrm{e}^{-\left(\frac{D}{2 C_{\mathrm{m}} x}\right)^{2}}}$, where $C_{\mathrm{m}}$ is the spreading coefficient, which

is 0.075 in this study, $U_{0}$ is the mean axial velocity along the jet centreline at distance $x$ from the jet exit and $D$ is the jet exit diameter
The distribution of the fluctuating velocity along the jet centreline normalized by the centreline mean velocity is presented in Fig. 12 for $50 \mu$ s and $100 \mu$ s delay times between the two recorded plasma images. Figure 12 presents the 'raw' measured turbulent intensity, the fluctuation intensity induced to the flow by the plasma of the LIPIV technique and the corrected turbulent intensity of the jet flow along the centreline. The comparison is with the jet flow from a round pipe with 86,000 Reynolds number (Xu and Antonia 2002) and the flow from a contoured jet exit with Reynolds number of $1.84 \times 10^{5}$ (Quinn 2006). According to Eq. (1), the 'raw' turbulence consists of the true flow

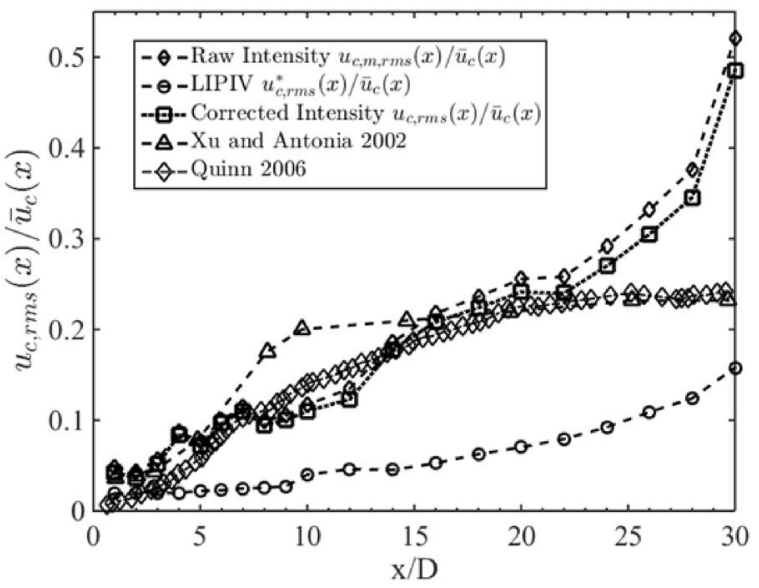

(a) $\Delta t=50 \mu \mathrm{s}$

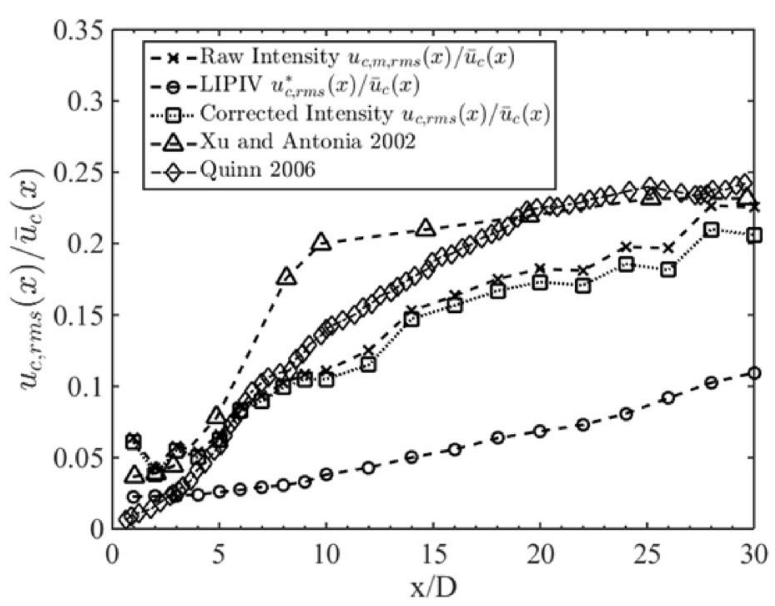

(b) $\Delta t=100 \mu \mathrm{s}$

Fig. 12 Normalized rms of axial velocity fluctuations along the centreline of the jet using time delay of $50 \mu \mathrm{s}$ (a) and $100 \mu \mathrm{s}$ (b) between the two plasma images (crosses for measured raw rms results, circles for uncertainty of the rms, squares for corrected rms results, triangles for $\mathrm{Xu}$ and Antonia, and diamonds for Quinn). The first plasma image was always at time delay of $1 \mu \mathrm{s}$ after the laser pulse initiation. Normalisation is based on the local centreline axial mean velocity $\bar{u}_{\mathrm{c}}(x)$ for each axial location 
field turbulence and the uncertainty due to the induced flow introduced by the plasma. If this uncertainty $u^{*}$ is independent of the flow field, the $u_{\mathrm{rms}}^{*}(x) / \bar{u}_{\mathcal{s}}(x)$ curve [where local centreline axial mean velocity is $u_{\mathrm{c}}(x)$ ] becomes an increasingly dominant source of uncertainty, as the mean velocity $u_{\mathrm{c}}(x)$ of the jet flow decays. After the subtraction of the $u_{\mathrm{rms}}^{*}(x) / \bar{u}_{\mathrm{c}}(x)$ component from the 'raw' intensity, (Eq. (3)), the corrected turbulent intensity can be obtained. The measured velocity fluctuations are lower than those in the literature (Xu and Antonia 2002; Quinn 2006) after $x / D>8$. This difference may be the result of the different geometrical configurations upstream of the jet exits used in the three studies as well as of the relatively poor spatial resolution of the LIPIV (about $5 \mathrm{~mm}$ ) as compared to the jet emanating from the $10 \mathrm{~mm}$ diameter jet exit. The present study utilized a pipe of length equal to 48 diameters upstream from the jet exit. In contrast, $\mathrm{Xu}$ and Antonia (2002) used a pipe with length equal to 118 inner diameters upstream of the jet exit and found an initial turbulent intensity of about $0.5 \%$ in the central region. Quinn (2006) employed a flow conditioning facility consisting of a large settling chamber fitted with honey-comb and mesh-wire screens and a three-dimensional contraction leading to $0.4 \%$ turbulent intensity at the exit plane. For the $50 \mu \mathrm{s}$ time delay in Fig. 12a, the corrected turbulent intensity becomes inconsistent with the measured values, especially at $x / D=25-30$, since the influence of the relatively large $u^{*}$ component on the measurement of the low local mean flow velocities was difficult to remove completely. The results for time delay of $100 \mu$ s between the plasma images in Fig. 12b provide the corrected turbulent intensity for the jet flow. Compared to turbulent intensity results of jet flows initiated from contraction and sharp-edged orifices (Quinn 2006), a jet flow initiated from a fully developed pipe flow has lower turbulent intensity, which has a magnitude of about 0.2 at $x / D=30$. This can be explained by the differences of the exit velocity profiles for different nozzles.

Figure 13 presents radial profiles of the mean and normalized mean axial velocity at different axial distances from the jet exit. The left side of each graph of Fig. 13 shows the results for $50 \mu$ s delay time between the plasma images and the corresponding right-hand side for $100 \mu \mathrm{s}$ delay time. The normalized radial profiles collapse well and can be approximated well by a Gaussian distribution. The normalisation of the radial profiles of the mean axial velocity is based on the centreline velocity at each axial location for the velocity and with the radius of the half width of the jet at each axial distance. (The half width of the jet is defined as the radial distance from the centreline of the jet, where the local mean velocity is equal to half of the local centreline mean velocity.). As shown by numerous studies (Namer and Ötügen 1988; Hu et al. 2000; Sorbe 2014), this normalisation also leads to self-similar

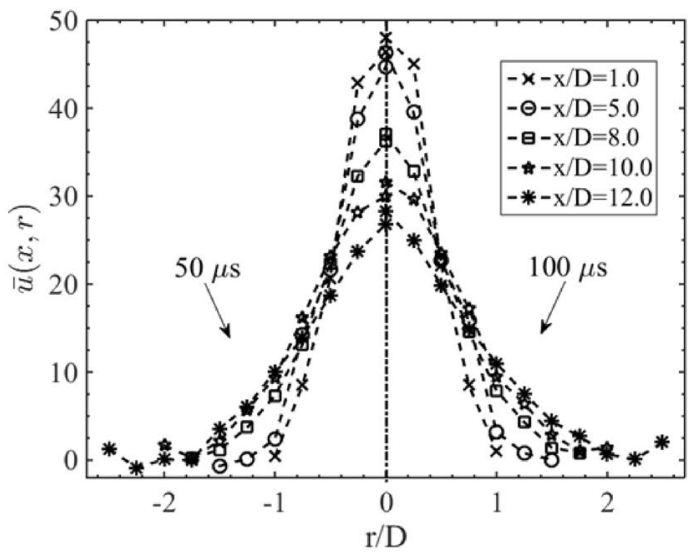

(a)

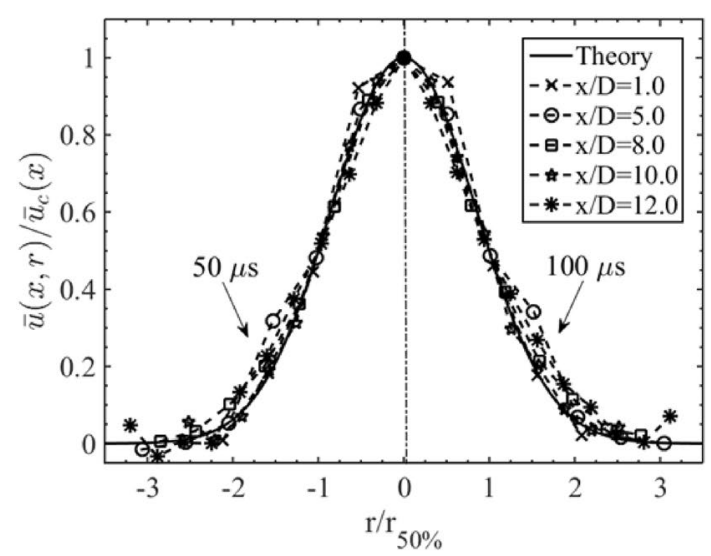

(b)

Fig. 13 Radial profiles of the mean (a) and normalized mean (b) axial velocity obtained at different axial locations from the jet exit $[x / D=1$ (crosses), 5 (circles), 8 (squares), 10 (pentagrams) and 12 (stars)]. The results are averaged over 300 instantaneous LIPIV velocity measurements at each point. The left and right sides of the figure show results for $50 \mu$ s and $100 \mu$ s delay times, respectively, between the recorded plasma images. The theoretical curve was plotted using a Gaussian profile normalized by the centreline velocity and the half jet width for each axial location

axial velocity profiles. This is confirmed from the findings of Fig. 13b, which provides some measure of confidence in the ability of the proposed LIPIV method to measure the flow velocity.

Figure 14 shows the rms of the axial velocity fluctuations as a function of the radial distance normalized by the jet exit diameter. Compared with hot-wire anemometry results at $x / D=3.0$ ( $\mathrm{Xu}$ and Antonia 2002), the normalized rms profiles, measured by LIPIV, appear to be wider. This is mainly attributed to the low resolution of LIPIV compared with the jet dimensions, leading to spatial averaging of the rms profiles. Nevertheless, the shape of the radial profiles for the rms of the axial velocity fluctuations is consistent with $\mathrm{Xu}$ and Antonia (2002), which again provides some measure of confidence in the ability 


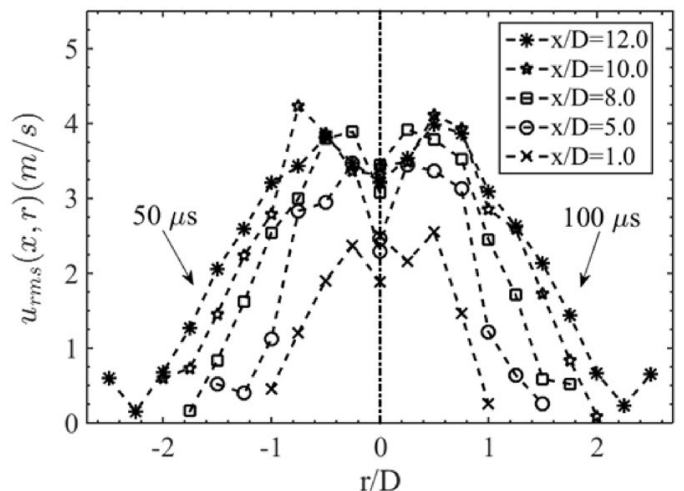

(a)

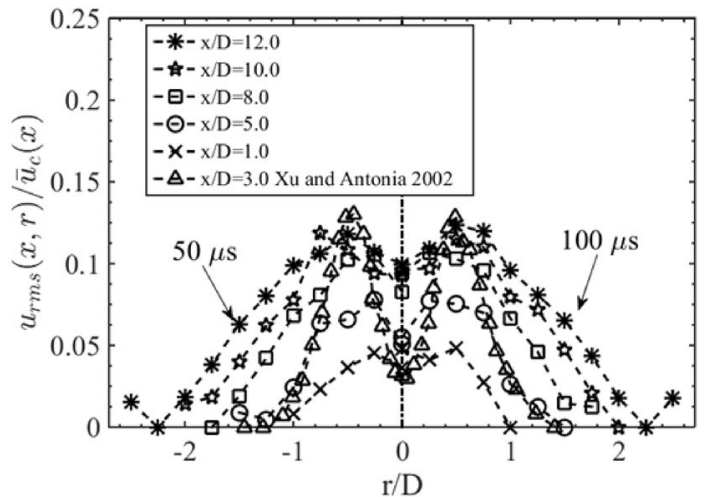

(b)

Fig. 14 Radial profiles of the rms (a) and normalized rms (b) of the axial velocity fluctuations obtained at different axial locations from the jet exit $[x / D=1$ (crosses), 3 (triangles), 5 (circles), 8 (squares), 10 (pentagrams) and 12 (stars)] averaged over 300 instantaneous velocity measurements at each point. The left and right hand sides of the graph show the results for $50 \mu$ s and $100 \mu$ s delay times between the recorded plasma images, respectively. Normalisation is based on the local centreline axial mean velocity $u_{\mathrm{c}}(x)$ for each axial location

of the proposed LIPIV method to obtain flow velocity measurements.

It is noted that in more complex flows than the currently reported jet flow, which have significant velocity component in the direction along the line of sight of the camera system, the displacement of the plasma due to the convection of the flow in this direction will introduce additional uncertainties. As is common with other existing velocity measurement techniques, e.g., particle image velocimetry, this issue can be addressed by introducing a second view that can observe the plasma temporal evolution from another direction. In this way, plasma tracking can be performed in three dimensions and provide measurements of three velocity components simultaneously. In addition, in flows where laser beam-stirring effects may occur, e.g., due to gas temperature gradients, the addition of a second view can also identify potential uncertainties. Such future extension of the LIPIV technique is possible.

\section{Conclusions}

In this paper, we presented a novel approach for point flow velocity measurements using the displacement of laserinduced plasma, which does not require the introduction of either 'seeding' particles or any other substances in the fluid. The laser-induced plasma was first investigated spatially, temporally and spectrally. The details of the image processing and the associated data processing for the measurement of the flow velocity using images of the temporal evolution of the laser-induced plasma were presented. An evaluation was performed of the accuracy of the velocity measurements and its dependence on the image processing method and on the selected delay time between the subsequent plasma images. The accuracy was evaluated in quiescent air surroundings. It was found that, using $100 \mu$ s time delay, LIPIV has a uncertainty of $-0.51 \pm 3.6 \mathrm{~m} / \mathrm{s}$ along the direction of the laser beam axis and $1.25 \pm 0.86 \mathrm{~m} / \mathrm{s}$ along the direction perpendicular to the laser beam axis. This uncertainty does not depend on the flow velocity, but on the behaviour of the induced plasma. The LIPIV was shown to be effective in quantifying the turbulent properties of a jet flow, although limitations exist. It should be noted that the method does not attempt to replace the currently available PIV or MTV methods that have higher spatial resolution and can measure more accurately and precisely the velocity characteristics when appropriate optical access is available. The properties of this technique can be summarized as follows.

LIPIV is based on widely available pulsed laser technology, without need of specific wavelength: two cameras are required. It is a calibration-free velocimetry technique, similar to the approach used for time of flight methods, like MTV. The method has the potential to be applied in harsh environments, such as combustion chambers, where it may be undesirable or difficult to introduce 'seeding' particles. It does not rely on any special molecule or atom species being present in the gas flows before the measurement, as most of the MTV methods do. In addition, it requires relatively small optical access, since the laser beam can be delivered and the induced plasma can be imaged through the same optical window. This is also an advantage for measurements in supersonic flows. The image processing is straightforward, compared to the spatial correlation algorithms of PIV or MTV approaches. The spectrum of the emitted light, presented in Fig. 3, from the plasma can also be used to measure local gas mixture composition, using the LIBS approach, simultaneously with flow velocity, using LIPIV.

This work is a preliminary investigation into the LIPIV technique and needs further investigations: for instance, the LIPIV technique generates a reactive plasma, a gas 
flow, and pressure waves, so that the technique is intrusive to a degree which depends on the flow under consideration. To this extent, LIPIV generates different and, arguably, greater intrusion than other 'seedless' techniques: whether the intrusion is acceptable depends on the circumstances of the flow. The demonstrated spatial resolution of the velocity measurements is about $5 \mathrm{~mm}$ and is a limitation owing to the size of the laser-induced plasma. However, higher resolution might be possible by reducing the plasma size to around $1 \mathrm{~mm}$, using lower laser pulse energy with lower signal-to-noise ratio. In contrast to particle image velocimetry, which is a planar technique, LIPIV is typically applied to obtain point measurements. However, it is possible to envisage an optical arrangement for simultaneous, multiple point measurements. In low speed flows (less than $10 \mathrm{~m} / \mathrm{s}$ ), the uncertainty of LIPIV is relatively large compared to the local flow speed. Finally, the interaction between the plasma and the flow should be further studied. In the complex environment of combustors and supersonic flows, the non-uniform distribution of temperature or pressure may deform the induced plasma, which may increase the uncertainty of LIPIV. Such applications require additional assessment.

Acknowledgements The authors would like to acknowledge the financial support of the China Scholarship Council (CSC)-Imperial College scholarship programme and of the Engineering and Physical Sciences Research Council (EPSRC) Grants EP/K021095/1 and EP/ M015300/1. YH and AMPKT would like to acknowledge discussions with Prof. Doug Greenhalgh.

Open Access This article is distributed under the terms of the Creative Commons Attribution 4.0 International License (http://creativeco mmons.org/licenses/by/4.0/), which permits unrestricted use, distribution, and reproduction in any medium, provided you give appropriate credit to the original author(s) and the source, provide a link to the Creative Commons license, and indicate if changes were made.

\section{References}

Akram M (1996) Two-dimensional model for spark discharge simulation in air. AIAA J 34:1835-1842

Alexander LG, Barron T, Comings EW (1953) Transport of momentum, mass, and heat in turbulent jets. University of Illinois, Engineering Experiment Station. Bulletin; no. 413. http://www.ideal s.illinois.edu/handle/2142/4429. Accessed 12 Dec 2016

Aziz TN, Raiford JP, Khan AA (2008) Numerical simulation of turbulent jets. Eng Appl Comput Fluid Mech 2:234-243

Bradley D, Sheppard CGW, Suardjaja IM, Woolley R (2004) Fundamentals of high-energy spark ignition with lasers. Combust Flame 138:55-77

Dors IG, Parigger CG (2003) Computational fluid-dynamic model of laser-induced breakdown in air. Appl Opt 42:5978-5985

Edwards CF, Rudoff RC (1990) Structure of a swirl stabilized spray flame by imaging laser Doppler velocimetry and phase Doppler anemometry. Proc Combust Inst 23:1353-1359
Elkins CJ, Alley MT (2007) Magnetic resonance velocimetry: applications of magnetic resonance imaging in the measurement of fluid motion. Exp Fluids 43:823-858

Elkins CJ, Markl M, Pelc N, Eaton JK (2003) 4D magnetic resonance velocimetry for mean velocity measurements in complex turbulent flows. Exp Fluids 34:494-503

Fellouah H, Pollard A (2009) The velocity spectra and turbulence length scale distributions in the near to intermediate regions of a round free turbulent jet. Phys Fluids 21:1-9

Ferioli F, Buckley S (2006) Measurements of hydrocarbons using laserinduced breakdown spectroscopy. Combust Flame 144:435-447

Forkey JN, Finkelstein ND, Lempert WR, Miles RB (1996) Demonstration and characterization of filtered Rayleigh scattering for planar velocity measurements. AIAA J 34:442-448

Hart RC, Balla RJ, Herring GC (2000) Simultaneous velocimetry and thermometry of air using nonresonant heterodyned laser-induced thermal acoustics. NASA ICASE report no. 2000-22, pp 1-6

Hong S, Speth RL, Shanbhogue SJ, Ghoniem AF (2013) Examining flow-flame interaction and the characteristic stretch rate in vortexdriven combustion dynamics using PIV and numerical simulation. Combust Flame 160:1381-1397

$\mathrm{Hu} \mathrm{H}$, Koochesfahani MM (2006) Molecular tagging velocimetry and thermometry and its application to the wake of a heated circular cylinder. Meas Sci Technol 17(6):1269-1281

Hu H, Saga T, Kobayashi T, Taniguchi N (2000) Simultaneous velocity and concentration measurements in a turbulent jet flow by using PIV-PLIF combined system. JSME KSME Therm Eng Conf 1-6

Joshi S, Olsen DB, Dumitrescu C et al (2009) Laser-induced breakdown spectroscopy for in-cylinder equivalence ratio measurements in laser-ignited natural gas engines. Appl Spectrosc 63:549-554

Kawahara N, Beduneau JL, Nakayama T et al (2006) Spatially, temporally, and spectrally resolved measurement of laser-induced plasma in air. Appl Phys B 86:605-614

Kiuchi M, Fujisawa N, Tomimatsu S (2005) Performance of a PIV system for a combusting flow and its application to a spray combustor model. J Vis 8:269-276

Kono M, Niu K, Tsukamoto T, Ujiie Y (1989) Mechanism of flame kernel formation produced by short duration sparks. Symp Int Combust Proc 22(1):1643-1649

Kotzagianni M, Yuan R, Mastorakos E, Couris S (2014) Laser-induced breakdown spectroscopy measurements in turbulent methane flames. In: 52nd AIAA aerospace sciences meeting. AIAA science and technology forum and exposition SciTech 2014, pp 1-14

Kravchik T, Sher E (1994) Numerical modeling of spark ignition and flame initiation in a quiescent methane-air mixture. Combust Flame 99:635-643

Lee SH, Do H, Yoh JJ (2016) Simultaneous optical ignition and spectroscopy of a two-phase spray flame. Combust Flame 165:334-345

Lempert WR, Jiang N, Sethuram S, Samimy M (2002) Molecular tagging velocimetry measurements in supersonic microjets introduction. AIAA J 40:5-10

Lyn DA, Einav S, Rodi W, Park J-H (1995) A laser-Doppler velocimetry study of ensemble-averaged characteristics of the turbulent near wake of a square cylinder. J Fluid Mech 304:285

Majd AE, Arabanian AS, Massudi R, Nazeri M (2011) Spatially resolved laser-induced breakdown spectroscopy in methane-air diffusion flames. Appl Spectrosc 65:36-42

McGann B, Carter CD, Ombrello T, Do H (2015) Direct spectrum matching of laser-induced breakdown for concentration and gas density measurements in turbulent reacting flows. Combust Flame 162:4479-4485

Mi J, Nathan GJ, Nobes DS (2001) Mixing characteristics of axisymmetric free jets from a contoured nozzle, an orifice plate and a pipe. J Fluids Eng 123:878 
Mi J, Kalt P, Nathan GJ, Wong CY (2007) PIV measurements of a turbulent jet issuing from round sharp-edged plate. Exp Fluids 42:625-637

Miles R, Cohen C, Connors J, Howard P, Huang S, Markovitz E, Russell G (1987) Velocity measurements by vibrational tagging and fluorescent probing of oxygen. Opt lett 12:861-863

Miziolek AW, Palleschi V, Schechter I (2008) Laser-induced breakdown spectroscopy (LIBS). Cambridge University Press, Cambridge

Molina A, Shaddix CR, Sickafoose SM et al (2005) Effect of temperature and $\mathrm{CO}_{2}$ concentration on laser-induced breakdown spectroscopy measurements of alkali fume. Spectrochim Acta Part B At Spectrosc 60:1103-1114

Morsy MH, Chung SH (2002) Numerical simulation of front lobe formation in laser-induced spark ignition of $\mathrm{CH}_{4} /$ air mixtures. Proc Combust Inst 29:1613-1619

Mulla IA, Chakravarthy SR, Swaminathan N, Balachandran R (2016) Evolution of flame-kernel in laser-induced spark ignited mixtures: a parametric study. Combust Flame 164:303-318

Namer I, Ötügen MV (1988) Velocity measurements in a plane turbulent air jet at moderate Reynolds numbers. Exp Fluids 6:387-399

Peterson B, Baum E, Bohm B et al (2013) High-speed PIV and LIF imaging of temperature stratification in an internal combustion engine. Proc Combust Inst 34:3653-3660

Pitz RW, Wehrmeyer JA, Ribarov LA, Oguss DA, Batliwala F, Debarber PA, Deusch S, Dimotakis PE (2000) Unseeded molecular flow tagging in cold and hot flows using ozone and hydroxyl tagging velocimetry. Meas Sci Technol 11:1259-1271

Prager J, Thiele M, Selle S, Riedel U (2003) Numerical simulation of spark ignition including ionization. VDI Berichte 28:721-724

Quinn WR (2006) Upstream nozzle shaping effects on near field flow in round turbulent free jets. Eur J Mech B Fluids 25:279-301

Raizer YP (1977) Laser-induced discharge phenomena. Consultants Bureau, New York

Ribarov LA, Wehrmeyer JA, Hu S, Pitz RW (2004) Multiline hydroxyl tagging velocimetry measurements in reacting and nonreacting experimental flows. Exp Fluids 37:65-74

Shi Z (2018) Local air-fuel ratio and gas velocity measurements in flames using LIBS. Ph.D. thesis. Imperial College, London

Shi Z, Hardalupas Y, Taylor AMKP (2016) Local equivalence ratio measurement in opposed jet flames of premixed and non-premixed methane-air using laser-induced breakdown spectroscopy. In: 18th International symposium on the application of laser and imaging techniques to fluid mechanics, Lisbon, 4-7 July 2018, pp 1-15

Sijtsema NM, Dam NJ, Klein-Douwel RJH, Meulen TJJ (2002) Air photolysis and recombination tracking: a new molecular tagging velocimetry scheme. AIAA J 40(6):1061-1064
Sislian JP, Jiang LY, Cusworth RA (1988) Laser Doppler velocimetry investigation of the turbulence structure of axisymmetric diffusion flames. Prog Energy Combust Sci 14:99-146

Sorbe J (2014) An experimental study in the near field of a turbulent round free jet. Dissertation, University of Gävle, 1-74

Stavropoulos P, Michalakou A, Skevis G, Couris S (2005) Quantitative local equivalence ratio determination in laminar premixed methane-air flames by laser induced breakdown spectroscopy (LIBS). Chem Phys Lett 404:309-314

Stevens EJ, Bray KNC, Lecordier B (1998) Velocity and scalar statistics for premixed turbulent stagnation flames using PIV. Symp Combust 27:949-955

Stier B, Koochesfahani MM (1999) Molecular Tagging Velocimetry (MTV) measurements in gas phase flows. Exp Fluids 26:297-304

Taylor GI (1950) The instability of liquid surface when accelerated in a direction perpendicular to their planes. Proc Lond Math Soc A 201:192

The MathWorks Inc (2014) Regionprops: documentation. https:// uk.mathworks.com/help/images/ref/regionprops.html\#buoogbr-1. Accessed 30 Jan 2017

Troiani G, Marrocco M, Giammartini S, Casciola CM (2009) Countergradient transport in the combustion of a premixed $\mathrm{CH}_{4} /$ air annular jet by combined PIV/OH-LIF. Combust Flame 156:608-620

Tropea C (1995) Laser Doppler anemometry: recent developments and future challenges. Meas Sci Technol 6:605-619

Uchiyama T, Kobayashi M, Iio S et al (2013) Direct numerical simulation of a jet issuing from rectangular nozzle by the vortex in cell method. 2013:321-330

Van Maaren A, De Goey LPH (1994) Laser Doppler thermometry in flat flames. Combust Sci Technol 99:105-118

Van Maaren A, Thung DS, De Goey LRH (1994) Measurement of flame temperature and adiabatic burning velocity of methane/air mixtures. Combust Sci Technol 96:327-344

Willert C, Jarius M (2002) Planar flow field measurements in atmospheric and pressurized combustion chambers. Exp Fluids 33:931-939

Xu G, Antonia R (2002) Effect of different initial conditions on a turbulent round free jet. Exp Fluids 33(5):677-683

Yaroshchyk P, Body D, Morrison RJS, Chadwick BL (2006) A semiquantitative standard-less analysis method for laser-induced breakdown spectroscopy. Spectrochim Acta Part B At Spectrosc 61:200-209

Publisher's Note Springer Nature remains neutral with regard to jurisdictional claims in published maps and institutional affiliations. 